\title{
Le traumatisme acoustique
}

$L$ 'oreille interne est un organe sensoriel complexe et fragile dont la fonction est de transformer les stimuli auditifs, mécaniques, en influx nerveux. Des traumatismes acoustiques, aigus et intenses ou modérés et chroniques, peuvent entraîner des déficits auditifs, temporaires (fatigue auditive) ou permanents. Les déficits permanents sont avant tout secondaires à des pertes de cellules ciliées externes qui, chez les mammifères, contrairement à ce qui se passe chez les oiseaux, ne régénèrent pas. Il existe au moins deux types de réflexes adaptatifs protégeant l'oreille interne contre une stimulation acoustique trop forte, l'un diminuant la transmission au niveau de l'oreille moyenne et l'autre modifiant, grâce à une innervation afférente, les propriétés intrinsèques de contractibilité des cellules ciliées externes. Certaines expériences laissent penser qu'une résistance acquise aux traumatismes sonores pourrait se développer à la suite d'un entraînement progressif au bruit.

\section{Armand Dancer}

\section{ADRESSE}

A. Dancer : docteur vétérinaire (B2-156), docteur de 3e cycle (spécialité : physique), docteur ès sciences naturelles, chef du groupe physiologie, maître de recherches. Institut franco-allemand de recherches de Saint-Louis, BP 24, 68301 Saint-Louis Cedex, France 'oreille interne est la cible des traumatismes sonores. C'est à l'intérieur de cet organe (figure 1, p. 358) que se trouvent les délicates structures sensorielles qui transforment les stimuli acoustiques en messages nerveux. Ces structures peuvent être détruites très rapidement par un son intense tout comme la rétine peut l'être par un rayon laser ou par l'observation imprudente du soleil.

Les vibrations transmises aux liquides de l'oreille interne, par l'intermédiaire de l'oreille externe et de l'oreille moyenne, provoquent des déplacements de la membrane basilaire qui est enroulée en spirale de la base au sommet de la cochlée et qui supporte l'organe de Corti [1] (figure 2, p. 359). Ces déplacements s'organisent sous la forme d'une onde propagée (travelling wave) dont l'enveloppe se déplace de la base vers l'apex de la cochlée. Pour les fréquences aiguës, l'amplitude de l'onde est maximale à la base tandis que pour les fréquences graves elle l'est à l'apex. Ce phénomène mécanique est à l'origine de la tonotopie : l'excitation maximale se localise en un point de la cochlée qui dépend de la fréquence, ce qui permet la discrimination des hauteurs des sons. Les mouvements de la membrane basilaire induisent des cisaillements des stéréocils des cellules ciliées et, par voie de conséquence, une série d'événements (mécaniques, électriques et biochimiques) qui constituent la transduction mécano-sensorielle et la première étape de l'analyse du signal acoustique. Les cisaillements des sté- 


\section{RÉFÉRENCES}

1. Sterkers O, Ferrary E, Tran Ba Huy P Production des liquides de l'oreille interne. médecine/sciences $1990 ; 6$ : 755-61.

2. Dallos P. The problem of linearity. In The Auditory Periphery. New York, London Academic Press, 1973 : 203-4.

3. Békésy G. Direct observation of the vibrations of the cochlear partition under a microscope. Acta Oto Laryngol 1952 ; 42 : 197-201.

4. ISO 1999. Acoustics. Determination of occupational noise exposure and estimation of noise-induced hearing impairment, 2nd ed. Genève : International Standardization Organization, 1990

5. Hohmann BW. Musique et troubles de l'ouïe. In : Sécurité dans les loisirs, réf. : 84001 f. Caisse Nationale d'Assurance Suisse, $1990: 16$

6. Davis $\mathrm{H}$. An active process in cochlear mechanics. Hearing Res 1983 ; 9 : 79-90. 7. Aran JM. L'amplificateur cochléaire médecine/sciences $1990 ; 6: 740-3$

8. Pujol R. Anatomie et physiologie de la cochlée. Arch Int Physiol Biochim 1989 ; 97 A51-78.

9. Tran Ba Huy P. Ototoxicité des aminoglycosides. médecine/sciences $1988 ; 4$ : 36-41.

10. Saunders JC, Canlon B, Flock A Changes in stereocilia micromechanics following overstimulation in metabolically blocked hair cells. Hearing Res 1986 ; 24 : 217-25

11. Liberman MC, Dodds LW. Acute ultrastructural changes in acoustic trauma : serial-section reconstruction of stereocilia and cuticular plates. Hearing Res $1987 ; 16$ : 45-64.

12. Pickles JO, Comis SD, Osborne MP Cross-links between stereocilia in the Guinea pig organ of Corti, and their possible relation to sensory transduction. Hearing Res 1984 ; $15: 103-12$.

13. Robertson D. Functional significance of dendritic swelling after loud sounds in the Guinea pig cochlea. Hearing Res 1983 ; 9 : 263-78.

14. Dancer A. Étude expérimentale des traumatismes acoustiques. In : Legouix JP ed. Physiologie et physiopathologie des récepteurs auditifs. Monographie du sćminaire du Collège de France. Groupe Audition du Groupement des Acousticiens de Langue Française, 1983 : 61-80

15. Campo $P$, Lataye $R$. Intermittent noise and equal energy hypothesis. In : Dancer A, Henderson D, Salvi R, Hamernik RP, eds. Noise-induced hearing loss. Philadelphia, Toronto : BC Decker, 1991: 595-606.

16. Henderson D, Hamernik RP. Asymptotic threshold shift from impulse noise. In Hamernik RP, Henderson D, Salvi R, eds New Perspectives in Noise. Induced Hearing Loss.

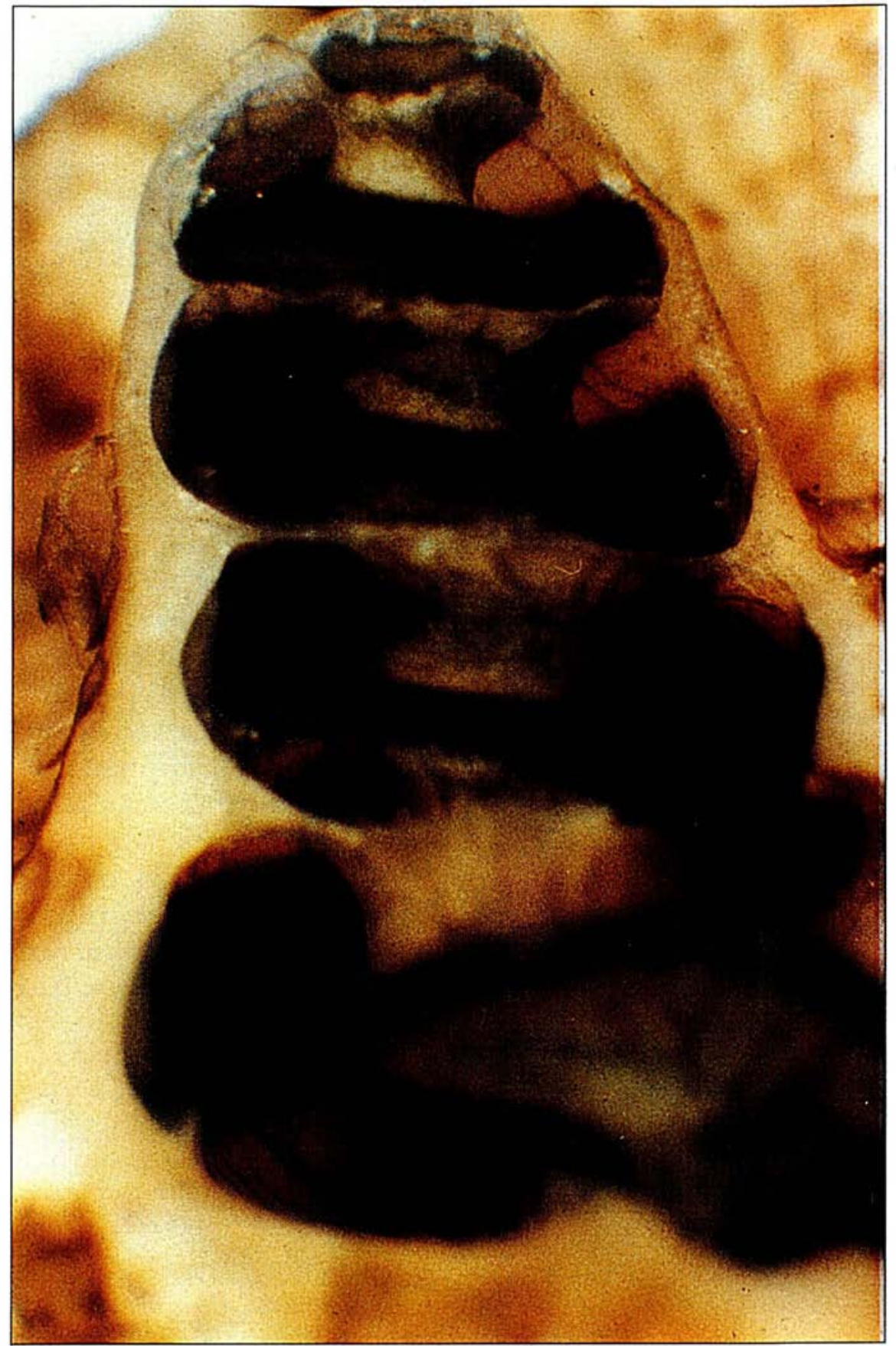

Figure 1. Cochlée de cobaye ouverte révélant la membrane basilaire enroulée en spirale. (Cliché Inserm, U. 229, Bordeaux.)

réocils provoquent en effet l'ouverture de canaux ioniques qui modifient la perméabilité des membranes cellulaires et qui permettent la pénétration des ions potassium à l'intérieur des cellules, entraînant ainsi leur dépolarisation. Cette dépolarisation provoque la libération de neu- romédiateur au pôle synaptique des sance aux influx nerveux qui vont parcourir les fibres afférentes du nerf auditif en direction des centres.

Au seuil de sensibilité auditive, l'amplitude des mouvements de la membrane basilaire et des stéréocils cellules ciliées internes et donne nais- 


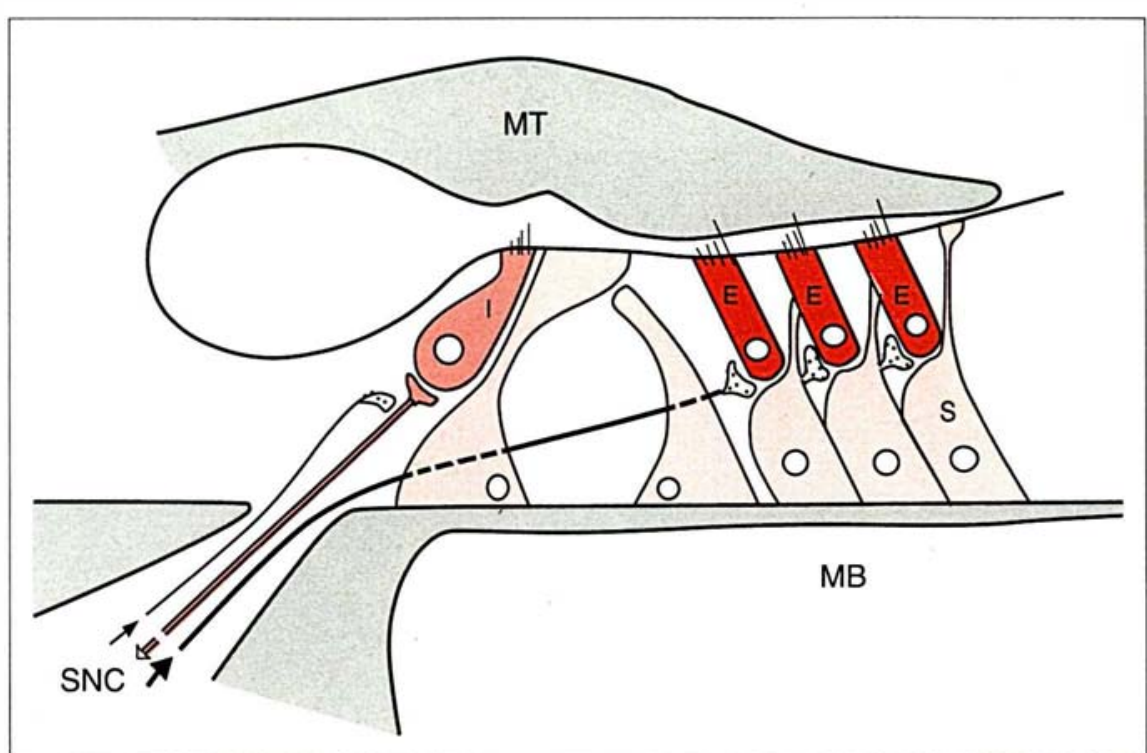

Figure 2. Représentation schématique d'une coupe de l'organe de Corti passant par une cellule ciliée interne (I) et trois cellules ciliées externes (E). Des cellules de soutien (S) relient les cellules ciliées à la membrane basilaire (MB) et la rangée la plus longue des cils des cellules ciliées externes est implantée dans la membrane tectoriale (MT). L'essentiel de l'innervation est également schématisé : fibre afférente prenant le message auditif de la cellule ciliée interne et l'amenant au système nerveux central (nerf auditif); fibre efférente latérale contrôlant le départ du message auditif ; fibre efférente médiane contrôlant les mouvements des cellules ciliées externes et donc le couplage entre la membrane tectoriale et la membrane basilaire. (Schéma Inserm, U. 254, Montpellier.)

est extrêmement faible. Selon Dallos [2], qui extrapole linéairement les résultats de von Békésy [3], l'amplitude serait inférieure à un picomètre $\left(10^{-2} \AA\right)$ : près d'un millionième du diamètre d'un stéréocil! Si l'on rapporte cette amplitude à un stéréocil de même hauteur que la tour Eiffel (!), le déplacement de son sommet, au seuil, ne serait que d'un dixième de millimètre environ.

Cependant, la gamme dynamique des stimuli sonores auxquels l'oreille peut être exposée est considérable (facteur $10^{6}$ au moins). Aux niveaux de stimulation très élevés, l'amplitude des déplacements des cils pourra donc être un million de fois plus grande et atteindre le même ordre de grandeur que celui des structures sensorielles (on observerait, par exemple, un déplacement de 100 mètres au sommet de notre tour sance de plus en plus inacceptable. Si l'on peut noter une amélioration de la protection de l'ouie en milieu industriel à la suite des diverses réglementations antibruit qui limitent à 90 ou à $85 \mathrm{dBA}^{*}$ le niveau auquel un individu peut cêtre exposé pendant une journée de travail de 8 heures et qui impose l'utilisation de protecteurs auditifs pour les expositions plus sévères [4], on constate dans le même temps une dégradation de l'audition chez les sujets les plus jeunes vraisemblablement à cause de l'impact des bruits de loisirs [5].

L'origine des déficits auditifs, leur évolution, les différences de susceptibilité d'un sujet à l'autre, les risques présentés par l'association entre le bruit et d'autres agents, les moyens de protection contre le bruit, les possibilités de traitement... sont des questions qui méritent d'être examinées en détail. Nous verrons que leur étude réserve parfois des surprises de taille du fait de l'avancée considérable des connaissances dans ce domaine depuis quelques années.

\section{Cellules ciliées et origine des déficits auditifs}

Les pertes auditives s'accompagnent généralement de la destruction d'une partie des cellules ciliées (au nombre de 15000 seulement à la naissance chez l'homme). Ces cellules sont incapables de se régénérer: toute destruction de ce petit contingent est donc définitive et cumulable et n'est, à l'heure actuelle, susceptible d'aucun traitement.

rapide (figure 3 , p. 360) : le traumatisme sonore est alors d'origine purement mécanique.

Les bruits peuvent aussi agir sur le métabolisme des cellules de l'organe de Corti ainsi qu'au niveau des synapses afférentes situées sous les cellules ciliées internes, surtout dans le cas d'expositions répétées et de longue durée.

Dans chacun de ces cas (traumatisme d'origine mécanique ou lésions d'origine métabolique), on assiste à une dégradation de la fonction auditive sous la forme de fatigue auditive et/ou de pertes auditives.

Le problème de santé publique posé par les effets des bruits sur l'audition est loin d'être résolu et est même ressenti actuellement comme une nuirence de $2.10^{-5} \mathrm{~Pa}$ (seuil d'audition a $1000 \mathrm{~Hz}$
* Le décibel $(d B)$ est une unité relative qui exprime sur une échelle logarithmique et par rapport à la réfé chez l'homme), le niveau de pression acoustique selon la formule

$$
20 \log \overline{2.10^{-5}}
$$

$(P$ : pression acoustique exprimée en pascals : Pa.) On parle alors de $d B S P L$ (sound pressure level). Pour tenir compte de la sensibilité de l'oreille humaine, sensibilité qui varie avec la fréquence et qui est maximale vers $3000 \mathrm{~Hz}$, on applique généralement à la mesure des bruits une pondération qui est fonction de la fréquence. La pondération la plus utilisée est la pondération (A) qui modifie le niveau des sons, au moins dans une certaine gamme de fréquences, d'après la fonction de transfert de l'ensemble oreille externeoreille moyenne de l'homme. 


\section{RÉFÉRENCES}

17. Botte MC, Baruch C, Dancer A. TTS as a function of exposure frequency. $J$ Acous tique $1990 ; 3: 53-7$

18. Kryter KD. The effects of noise on man. New York, London : Academic Press, $1970: 633$

19. Burns W, Robinson DIV. Hearing and Noise in Industry. London: Her Majesty' Stationery Office, 1970.

20. Buck K, Franke R. Can TTS be an indicator for individual susceptibility to PTS ? In : Salvi R, Henderson D, Hamernik RP, Colletti V, eds. Basic and applied aspects of noise-induced hearing loss. Nato Asi series, series A, Life Sciences, vol. 111, New York: Plenum Press, 1986 : 411-56. 21. Pujol R. Neurobiologie de la cochlće. médecine/sciences $1990 ; 6: 456-63$.

22. Dulon D, Aran JM. Aspects cellulaires et moléculaires de la transduction mécanosensorielle dans l'oreille interne. médecine/sciences $1990 ; 6: 744-54$

23. Liberman MC. Rapid assessment of sound-evoked olivocochlear feedback : suppression of compound action potentials by controlateral sound. Hearing Res 1989 ; 38 : 47-56.

24. Dancer A. Possibilités d'application à l'homme des résultats des ćtudes des effets des bruits sur l'audition réalisćes chez l'animal. Acustica 1981; 48 : 239-46.

25. Décory L, Dancer A, Aran JM. Species differences and mechanisms of damage. In : Dancer A, Henderson D, Salvi R, Hamernick RP, eds. Nose-induced hearing loss. Philadelphia-Toronto : B.C. Decker, 1991 91-108.

26. Boettcher FA Bancroft B, Gratton MA. Interaction of noise and other ototraumatic agents : recent advances. In : Dancer A, Henderson D, Salvi R, Hamernik RP, eds. Noise-induced hearing loss. Philadelphia, Toronto : BC Decker, 1991 : 220-36. 27. Aran JM, Hiel H, Hayashida T, Erre $\mathrm{JP}$, et al. Noise and aminoglycoside ototoxicity. In : Dancer A, Henderson D, Salvi R, Hamernik RP, eds. Noise-incluced hearing loss. Philadelphia, Toronto : BC Decker, 1991 . 237-46.

28. Campo P, Cnockaert JC. Risques auditifs encourus par le fotus chez la fermme enceinte exposće au bruit. Cahiers el Notes documentaires de l'INRS $1989 ; 137$ : 633-7. 29. Rajan R. Protective effects of the cochlear efferents on temporary threshold shifts in the Guinea pig. In : Dancer A, Henderson D, Salvi R, Hamernik RP, eds. Noiseinduced hearing loss. Philadelphia, Toronto BC Decker, 1991 : 562-83

30. Brundin L, Flock A, Canlon B. Soundinduced motility of isolated cochlear outer hair cells is frequency-specific. Nature 1989 $342: 814-6$

31. Canlon B, Borg E Current findings on the functional and morphological aspects of the training phenomenon. In : Dancer $\mathrm{A}$, Henderson D, Salvi R, Hamernik RP, eds. Noise-induced hearing loss. Philadelphia and Toronto : BC Becker, 1991: 629-43 32. Rubel EW. Regeneration of hair cells in the avian inner ear. In : Dancer A, Henderson D, Salvi R, Hamernik RP, eds. Noise-induced hearing loss. Philadelphia,
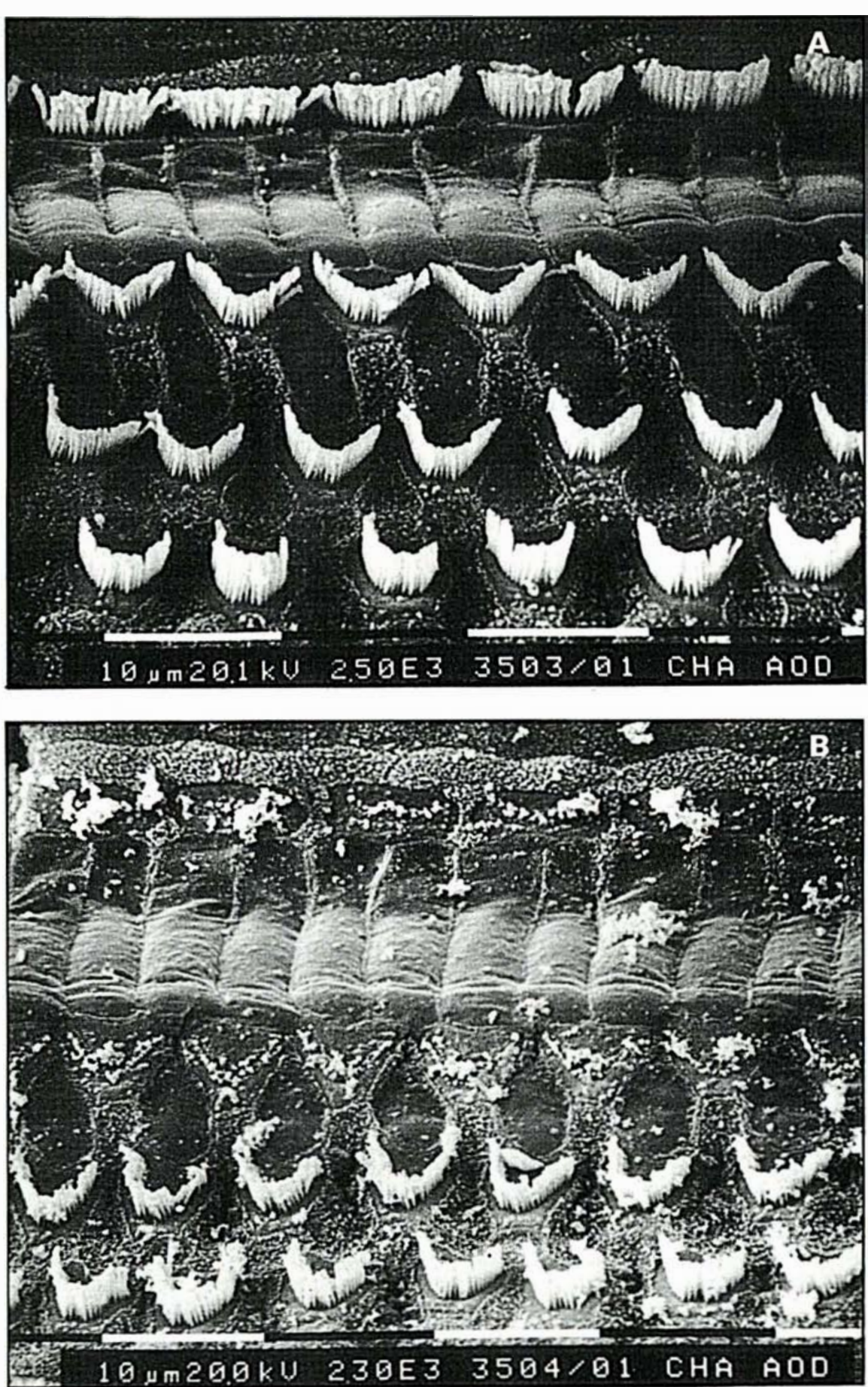

Figure 3. Vue d'un élément de l'organe de Corti chez le chat. (A) cochlée normale, (B) après exposition à un son pur de $8 \mathrm{kHz}$ à $120 \mathrm{~dB}$ pendant 20 minutes. On aperçoit nettement les touffes de cellules ciliées désorganisées. 
Il existe deux types de cellules ciliées : cellules ciliées internes et cellules ciliées externes, dont les rôles sont fort différents :

- les cellules ciliées internes (CCI) sont des mécanorécepteurs qui transforment les signaux produits par les vibrations acoustiques en messages envoyés au système nerveux central, mais elles sont incapables d'assurer à l'oreille sa sensibilité au seuil ainsi que sa remarquable sélectivité en fréquences (l'oreille des mammifères est capable de détecter des variations de pression de $10^{-5} \mathrm{~Pa}$, ou $-6 \mathrm{~dB}$ SPL, et de distinguer des changements de fréquence de 3,5 pour 1000 : intervalle 200 fois plus petit qu'une octave) ;

- les cellules ciliées externes (CCE) n'envoient pas de message auditif au système nerveux central. Elles amplifient sélectivement les vibrations mécano-acoustiques près du seuil (d'un facteur 100 environ, soit $40 \mathrm{~dB}$ ) et permettent ainsi (vraisemblablement par couplage micromécanique via la membrane tectoriale) l'excitation des cellules ciliées internes. Les cellules ciliées externes peuvent produire davantage d'énergie qu'elles n'en reçoivent du milieu extérieur et se contracter activement à des fréquences de plusieurs kilohertz! C'est ce que Davis [6] a surnommé l'" amplificateur cochléaire " [7]. Cet " amplificateur " est d'ailleurs à l'origine des otoémissions acoustiques spontanées ou provoquées [8]. Si les cellules ciliées externes et leurs stéréocils sont lésés, seuls les phénomènes hydromécaniques passifs subsistent et ils ne permettent qu'une analyse grossière des vibrations sonores. Or ce sont les cellules ciliées externes qui sont les plus susceptibles au traumatisme sonore (ainsi qu'à bon nombre d'autres facteurs : anoxie, antibiotiques ototoxiques [9], chimiothérapies antitumorales...) et qui sont les premières détruites. Très schématiquement, on peut dire que la destruction des touffes ciliaires des CCE entraîne une élévation des seuils de l'ordre de $40 \mathrm{~dB}$, l'apparition du phénomène de recrutement (qui correspond essentiellement à une compression de la gamme dynamique de l'oreille) et des difficultés de compréhension de la parole surtout en ambiance bruyante (difficultés liées à la détérioration de la sélectivité en fréquence).

Atteintes ciliaires. Une attention toute particulière a été portée par plusieurs auteurs aux propriétés micromécaniques des stéréocils des CCE. Saunders et al. [10] ont observé que leur raideur diminuait à la suite d'une surstimulation acoustique. Liberman et Dodds [11] ont pu montrer que la fatigue auditive était corrélée, sur le plan anatomique, à une diminution de la longueur des racines des cils. Ces modifications sont réversibles et leur récupération semble être sous la dépendance de phénomènes métaboliques. Une autre corrélation anatomique de la fatigue auditive concerne les connexions existant entre les cils. L'hypothèse de leur intervention dans les processus de transduction a été proposée par Pickles et al. [12]. La rupture de ces structures après l'exposition au bruit a pu être constatée en microscopie électronique à balayage. Elle entraînerait une diminution des contraintes provoquant l'ouverture des canaux ioniques, du nombre de canaux actifs et donc de la dépolarisation de ces cellules.

En résistance des matériaux, on sait qu'au-delà d'une certaine limite la relation entre l'allongement et la contrainte devient non linéaire, puis la rupture de la structure survient. Il doit en être de même pour les cils : pour les niveaux très élevés, bruits impulsionnels... une seule déflexion dépassant la limite de rupture serait suffisante à détruire la structure alors que lors de l'exposition à des niveaux plus modérés, la limite d'élasticité n'étant pas dépassée, la durée de l'exposition jouerait un rôle très important et amènerait des ruptures par fatigue (lorsqu'un matériau est soumis à un très grand nombre de cycles de déformation, ce qui est le cas de la stimulation acoustique).

Atteintes cellulaires. En dehors des lésions ciliaires, de nombreuses autres altérations consécutives à l'exposition au bruit ont été décrites.

Des altérations présynaptiques : il existe au niveau des cellules ciliées des modifications ultrastructurales concomitantes de l'exposition au bruit (prolifération et vacuolisation du réticulum endoplasmique, gonflement des mitochondries, accumulation des lysosomes) [8].
Des altérations post-synaptiques : Robertson [13] a décrit un gonflement des dendrites afférentes (sous les cellules ciliées internes) immédiatement après l'exposition à un son pur. Ce phénomène est probablement la conséquence d'une quantité trop importante de messages synaptiques envoyés depuis les cellules ciliées internes vers ces dendrites. La présence d'un excès de neurotransmetteur glutamatergique dans l'espace intersynaptique peut en effet avoir un effet toxique.

Il semble néanmoins que ce soit surtout le degré de gravité des différents types d'altération des stéréocils qui détermine le caractère temporaire ou définitif des modifications fonctionnelles.

\section{Fatigue auditive et pertes auditives}

La fatigue auditive (ou élévation temporaire des seuils : ETS) se caractérise par une diminution de la sensibilité auditive qui persiste pendant un temps limité après la fin de la stimulation. L'étude de la récupération permet de distinguer plusieurs types de fatigue [14] :

- la fatigue à court terme, qui nécessite moins de deux minutes pour se dissiper. Le maximum des élévations de seuil se situe à la fréquence d'exposition ;

- la fatigue à long terme : ce type de fatigue est caractérisé par une durée de récupération supérieure à deux minutes mais inférieure à seize heures (limite arbitraire qui a été choisie au cours d'études sur les bruits industriels). La fatigue auditive est fonction de l'intensité, de la durée et de la fréquence de la stimulation. Elle est aussi fonction des conditions de présentation de la stimulation : une dose donnée de bruit appliquée sous forme intermittente est moins dangereuse pour l'audition [15].

L'amplitude des ETS augmente en moyenne de $6 \mathrm{~dB}$ chaque fois que celle de la stimulation double. A partir d'un certain niveau (niveau critique), cette augmentation s'accélère très fortement. Ce phénomène est particulièrement important dans le cas de l'exposition aux bruits impulsionnels. Au-delà d'une certaine durée d'exposition, les ETS atteignent une valeur asymptotique qui est d'autant plus 

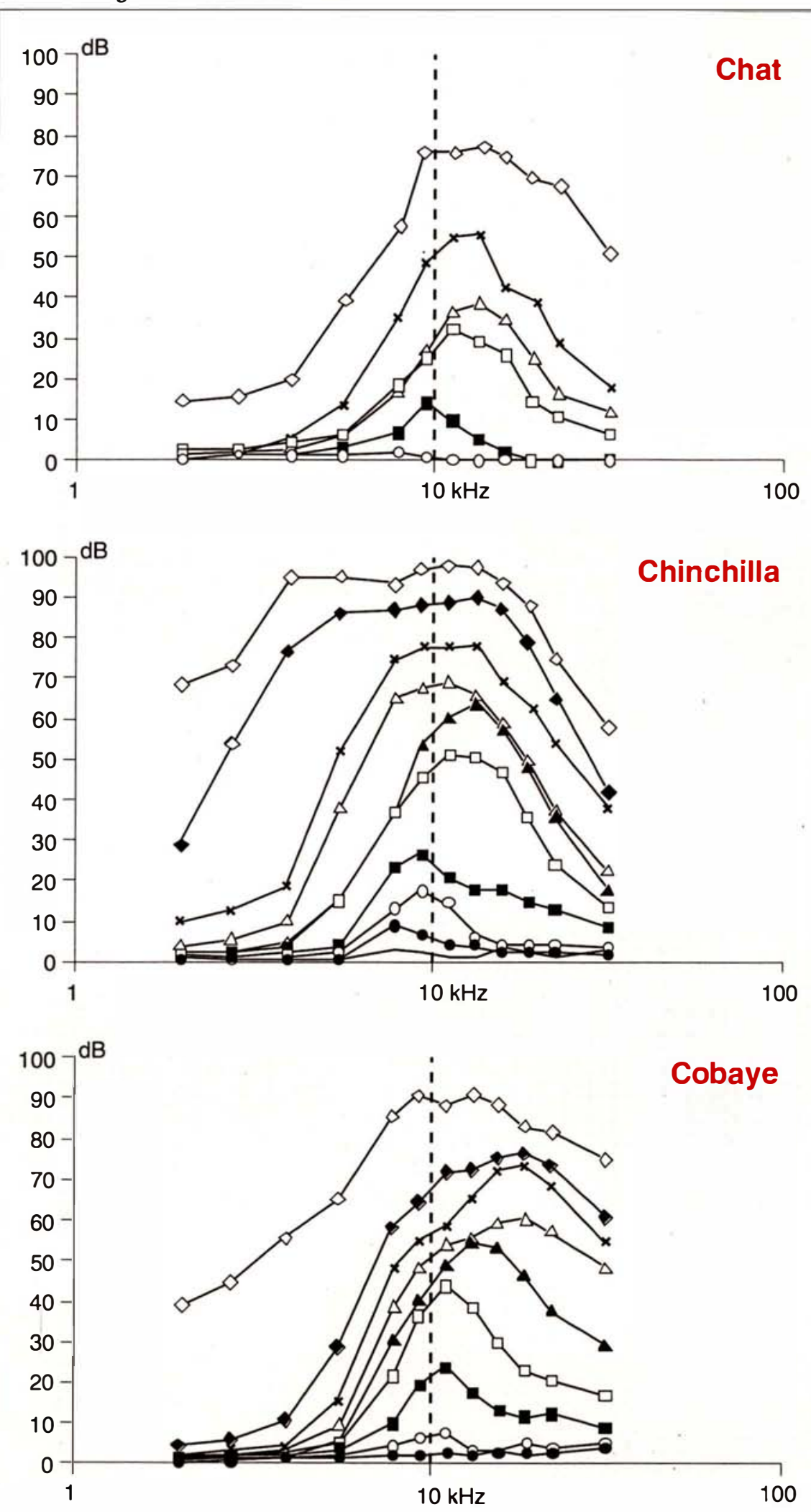

Niveaux de stimulation:

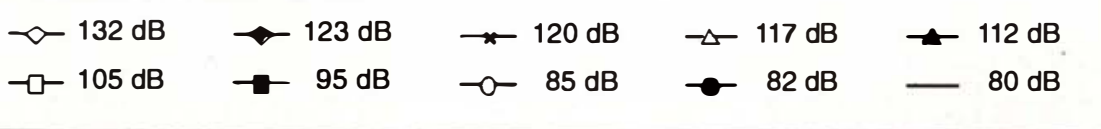

Figure 4. Chez le chat, le chinchilla et le cobaye, les amplitudes des élévations de seuils mesurées en décibels (en ordonnées) sont représentées en fonction de la fréquence audiométrique len abscisses, en kilohertz), 20 minutes après I'exposition à un son pur de $8 \mathrm{kHz}$ présenté, pendant 20 minutes, à des niveaux allant de 80 à 132 dB SPL. On remarque que l'amplitude des élévations de seuils augmente progressivement avec celle de la stimulation mais aussi que leur maximum se déplace progressivement vers des fréquences de plus en plus élevées (11-12 kHz) par rapport à la fréquence de stimulation (8 $\mathrm{kHz}$ ).

élevée que le niveau de la stimulation est plus fort [16]. Du fait de la fonction de transfert de l'oreille externe et de l'oreille moyenne, les basses fréquences sont beaucoup mieux tolérées que les fréquences moyennes et élevées : l'orcille est la plus susceptible aux fréquences pour lesquelles sa sensibilité est la meilleure. C'est en effet pour ces fréquences que l'énergie acoustique est transmise de façon optimale du milieu extérieur à l'oreille interne : voilà l'explication principale du fameux " trou à $4000 \mathrm{~Hz}$ " caractéristique des surdités professionnelles et bien connu de tous les cliniciens. Les effets du niveau et de la durée de la stimulation sont interdépendants. On a donc supposé que l'importance des déficits auditifs (et en particulier des EPS, élévation permanente des seuils) était liée à la quantité d'énergie acoustique reçue par l'oreille. C'est cette hypothèse qui a servi de base à l'établissement de normes internationales [4]. Il ne faut cependant pas considérer la mesure de l'énergie acoustique comme une solution universelle car, dans de nombreux cas, le principe d'isoénergie est pris en défaut : nous en avons vu un exemple pour les bruits intermittents. Il faut décrire ici un phénomène surprenant que l'on observe principalement à la suite de l'exposition à des sons purs et qui pose toute une série de questions fondamentales. On observe généralement que le maxi- 


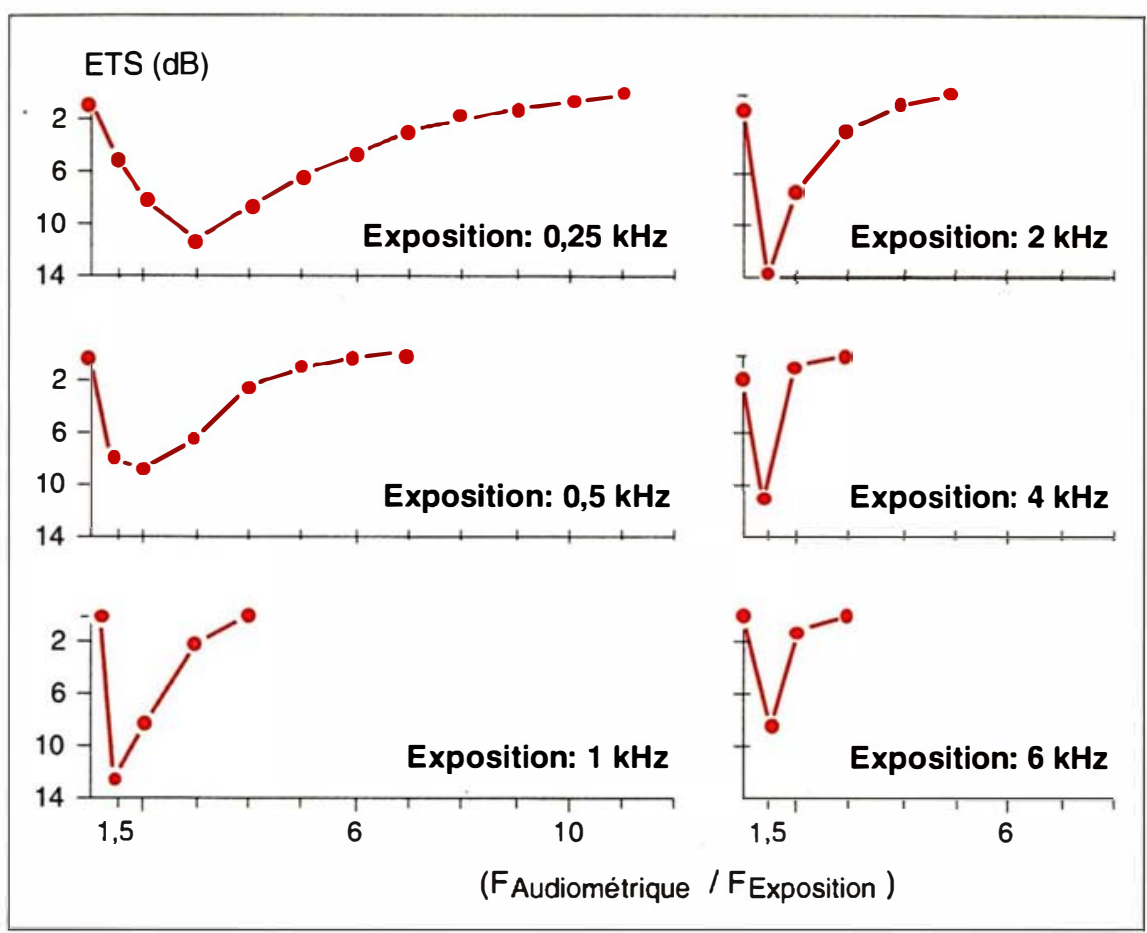

Figure 5. Élévations moyennes temporaires des seuils (ETS en dB) mesurées chez l'homme et représentées en fonction du rapport (en abscisses) entre la fréquence audiométrique et la fréquence d'exposition au son pur fatigant (pour 6 fréquences d'exposition différentes) (selon Botte et al., 1990). On remarque que les ETS sont maximales à des fréquences supérieures d'une demi-octave à la fréquence de stimulation et que ce décalage tend à augmenter lorsque la fréquence de stimulation est très basse : à $500 \mathrm{~Hz}$ et plus encore à $250 \mathrm{~Hz}$.

mum des ETS se décale progressivement vers les fréquences supérieures à celle de la stimulation fatigante au fur et à mesure que le niveau de cette dernière augmente (figure 4). Un sujet exposé à un son pur de $2000 \mathrm{~Hz}$ développe une fatigue auditive qui est maximale à $3000 \mathrm{~Hz}$ environ: c'est ce que l'on appelle communément le phénomène de décalage d'une demioctave [17] (figure 5) ! Ce phénomène a toujours intrigué les expérimentateurs. Il peut être expliqué, selon Davis [6], par les phénomènes actifs produits par les cellules ciliées externes. Cependant, aux niveaux plus élevés, le maximum des lésions ciliaires se déplace vers la fréquence de stimulation (figure 6, p. 364) et, plusieurs semaines après l'exposition, le décalage entre les élévations de seuil res- lesquelles s'établissent les pertes auditives chez l'homme, on a voulu utiliser les mesures de fatigue auditive (ETS, élévation temporaire des seuils) comme critère de susceptibilité aux pertes auditives (EPS). Kryter [18], en particulier, a émis l'hypothèse selon laquelle les EPS qui sont provoquées par des expositions répétées quotidiennement ne peuvent pas être plus importantes que les ETS mesurées deux minutes après la fin d'une exposition $\left(\mathrm{ETS}_{2 \mathrm{~min}}\right)$ et que des $\mathrm{ETS}_{2 \text { min }}$ égales induisent des risques égaux d'EPS. Burns et Robinson [19] ont recherché, chez des travailleurs de l'industrie, une corrélation entre les pertes auditives existantes et la fatigue auditive consécutive à une journée de travail ; ils ont suggéré que les ETS résultant d'une exposition journalière sont quantitativement similaires aux EPS résultant d'une même exposition pendant dix ans. Cependant il serait du plus grand intérêt de répondre à la question suivante : les individus qui présentent des ETS supérieures à la moyenne développeront-ils également des EPS supérieures à la moyenne ? Sur ce point, l'étude de Burns et Robinson s'est soldée par des résultats très peu significatifs. Etant donné que, comme nous l'avons vu plus haut, la fatigue auditive et les pertes auditives semblent induites par des mécanismes qui ne sont pas directement comparables, cela rendrait vaine toute tentative de prédiction des EPS à partir de la mesure des ETS chez un sujet donné.

tantes et la fréquence de stimulation est très réduit : les lésions ciliaires et les pertes cellulaires sont alors situées dans les zones de fréquence correspondant aux pertes auditives. Il est ainsi permis de penser que la fatigue et les pertes auditives participent de mécanismes différents, ce qui, comme nous le verrons plus loin, a des conséquences très importantes.

Les pertes auditives (EPS, élévation permanente des seuils) sont caractérisées par leur irréversibilité. On a cherché à établir des relations entre les paramètres physiques des bruits et les lésions. Étant donné la variabilité des atteintes auditives que l'on peut observer d'un individu à l'autre à la suite d'expositions sonores analogues et la quasi-impossibilité qu'il y a à étudier expérimentalement les conditions dans

\section{Différences de susceptibilité aux bruits}

L'importance de la variation interindividuelle de la susceptibilité de l'homme vis-à-vis du traumatisme acoustique a été reconnue depuis des décennies. Howell [voir Buck et Franke 20] a répertorié tous les tests visant à dépister les individus particulièrement sensibles :

- évaluation de l'état de santé général: une bonne fonction cardiovasculaire, un apport suffisant d'oxygène, un taux d'agrégabilité plaquettaire peu élevé et surtout une faible viscosité sanguine semblent limiter l'importance des ETS et/ou faciliter leur récupération ; 


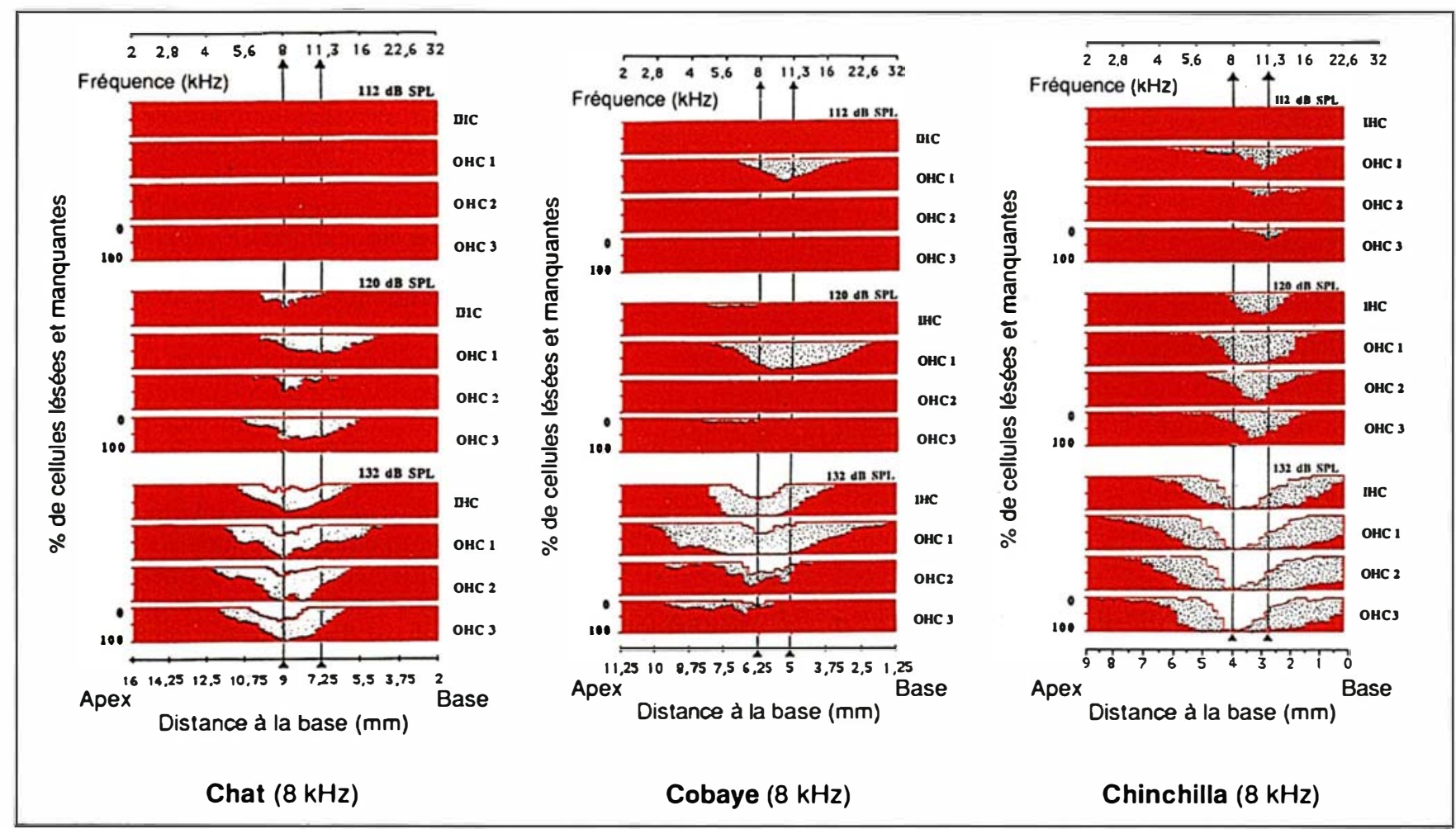

Figure 6. Cochléogrammes moyens obtenus chez le chat, le cobaye et le chinchilla à la suite d'une exposition à un son pur de $8 \mathrm{kHz}$ pendant 20 minutes à des niveaux de 112, 120 et $132 \mathrm{~dB}$ SPL. Les cellules intactes sont représentées en noir, les cellules lésées en grisé et les cellules détruites en blanc. On remarque que les premières lésions apparaissent sur la première rangée des cellules ciliées externes à un emplacement de fréquence caractéristique supérieure à celui correspondant à la fréquence de stimulation (flèches). En revanche, aux niveaux les plus élevés, les destructions cellulaires sont situées à un emplacement correspondant à la fréquence de stimulation. IHC : inner hair cells (en français, $\mathrm{CCl}$ : cellules ciliées internes). OHC : outer hair cells (en français : cellules ciliées externes). OHC1 correspond à la première rangée de cellules ciliées externes, OHC2 à la deuxième rangée et OHC 3 à la troisième rangée.

- couleur des yeux : un taux élevé de mélanine semble correspondre à une résistance élevée de la cochlée aux bruits :

- la mesure du réflexe acoustique de l'oreille moyenne : une bonne corrélation a été observée entre les différents paramètres liés au réflexe acoustique et les ETS consécutives à l'exposition aux bruits;

- influence du système efférent : les cellules ciliées externes sont innervées par le faisceau efférent (figure 2) [21]. Ces cellules contiennent des protéines contractiles et, en plus des mouvements rapides que nous avons décrits précédemment (mécanismes actifs rapides), sont capables de mouvements plus lents, dépendants de l'ATP, contrôlés en partie par les tes [22]. Liberman [23] a proposé que la susceptibilité interindividuelle au bruit soit liée à l'efficacité plus ou moins grande de la commande nerveuse efférente qui induirait des déplacements relativement lents mais de longue durée des structures sensorielles et les protégeraient en limitant leur gamme dynamique.

Les études concernant les effets des bruits sur l'audition dans différentes espèces animales ont permis d'établir des relations entre les paramètres de la stimulation acoustique, les modifications de la fonction auditive et les atteintes morphologiques de l'oreille interne.

On a ainsi pu montrer qu'une stimulation acoustique donnée pouvait entraîner des atteintes auditives très différentes selon l'espèce animale étu- diée : une exposition à un son pur de $1 \mathrm{kHz}$ à $120 \mathrm{~dB}$ SPL pendant 12 minutes produit, chez le chinchilla, des déficits auditifs supérieurs à ceux observés chez le singe écureuil après 12 heures d'exposition au même stimulus. Les relations quantitatives établies pour une certaine espèce ne sont donc pas directement transposables à une autre espèce et il est en particulier impossible de tirer, des études réalisées chez l'animal, des résultats quantitatifs applicables à l'homme [24]. Décory et al. [25] ont cherché à savoir si les différences interspécifiques de susceptibilité au bruit de l'organe périphérique de l'audition étaient dues:

- à la façon dont le stimulus acoustique présent dans le milieu extérieur est transmis à l'organe cible : l'oreille 
interne (c'est-à-dire essentiellement aux caractéristiques de la fonction de transfert de l'oreille externe et de l'oreille moyenne de chaque espèce) ;

- à la susceptibilité au bruit, propre à chaque espèce, de l'oreille interne ; - à une influence combinée de ces deux facteurs.

L'étude a été conduite chez le chat, le chinchilla et le cobaye. Il apparaît qu'une grande partie des différences interspécifiques de susceptibilité au bruit sont dues aux conditions de transmission des stimuli acoustiques jusqu'à l'entrée de l'oreille interne.

\section{Potentialisation des déficits auditifs par d'autres agents}

Le traumatisme acoustique n'est pas entièrement déterminé par les paramètres de la stimulation et peut être notoirement amplifié par l'action conjuguée d'autres agents [26]. Les plus connus sont : l'anoxie, les antibiotiques du type aminoglycosique [9], les hypomagnésémies sévères, certaines chimiothérapies antitumorales... On a pu montrer que la plupart de ces agents, employés isolément, lèsent préférentiellement les cellules ciliées externes de la base de la cochlée, tout comme les stimuli sonores trop intenses. De nombreuses études chez l'animal [27] ont montré que l'administration d'antibiotiques ototoxiques potentialise l'effet des bruits (et vice versa) lorsque ces agents sont administrés à des doses proches des doses nocives de chacun d'eux pris isolément.

Enfin il faut mentionner qu'il existe, au cours du développement de l'oreille des mammifères, une période critique qui correspond à l'entrée en fonction de l'organe et pendant laquelle la cochlée est extrêmement sensible aux agressions sonores. Chez l'homme, cette période a lieu in utero à partir du sixième mois et il existe, à l'heure actuelle, une controverse quant au danger que pourrait représenter pour le fotus l'exposition de la mère aux bruits [28]. Les conditions de transmission des bruits du milieu extérieur à l'oreille interne du fœtus sont très mal connues, mais il existe en tout état de cause une atténuation très importante du fait de la mauvaise adaptation d'impédance qui existe entre le milieu aérien et les milieux liquides dans lesquels baigne le fœetus (dont l'oreille moyenne est également remplie de liquide). De ce fait, il ne semble pas que des précautions particulières soient nécessaires, excepté lors d'expositions de très haut niveau à des fréquences inférieures ou égales à $500 \mathrm{~Hz}$ environ, au cours des trois derniers mois de la grossesse.

\section{Réflexes \\ de protection acoustique}

L'oreille dispose de mécanismes qui lui permettent, sous certaines conditions, de se protéger contre les bruits trop intenses. Nous parlerons ici, de manière délibérément provocatrice, des réflexes de protection acoustique. En effet, si le réflexe de protection de l'oreille moyenne (réflexe acoustique ou réflexe stapédien chez l'homme) est bien connu, il semble qu'il existe, au niveau de l'oreille interne, un autre réflexe d'importance au moins comparable au premier et qui a pour origine les cellules ciliées externes de l'organe de Corti.

L'application d'un stimulus sonore de niveau élevé à l'entrée de l'oreille provoque la contraction bilatérale des muscles de l'oreille moyenne : tenseur du tympan et stapédien (chez l'homme, seul le stapédien se contracte). Les muscles de l'oreille moyenne peuvent avoir des rôles multiples : extension de la gamme dynamique de l'oreille, amélioration de la localisation des sources sonores, diminution des résonances de l'oreille moyenne, régulation de la pression aérienne dans l'oreille moyenne et de la pression liquidienne dans l'oreille interne, mais surtout : protection de l'oreille interne contre les stimuli acoustiques de fort niveau. Chez l'homme, dans la gamme des fréquences comprises entre 250 et $4000 \mathrm{~Hz}$, le seuil du réflexe stapédien se situe à environ $80 \mathrm{~dB}$ au-dessus du seuil d'audition et son amplitude croît avec le niveau de la stimulation (de $0,6 \mathrm{~dB} / \mathrm{dB}$ environ). La latence de ce réflexe est de $150 \mathrm{~ms}$ au seuil et de $25 \mathrm{~ms}$ à $35 \mathrm{~ms}$ aux forts niveaux. La contraction du muscle stapédien peut être réalisée de façon volontaire par certains sujets sans qu'il soit nécessaire d'utiliser un son fort. Chez l'homme et chez les animaux, la contraction des muscles de l'oreille moyenne réduit (de $10 \mathrm{~dB}$ environ) la transmission des sons à l'oreille interne aux fréquences inférieures à la fréquence de résonance principale de l'oreille moyenne. Étant donné sa latence, ce réflexe ne peut protéger l'oreille des bruits impulsionnels présentés isolément. En revanche, dès que le rythme de présentation est supérieur à 2 ou 3 par seconde, on commence à pouvoir mettre en évidence une diminution de la fatigue auditive. La fatigabilité du réflexe ne lui permet pas de protéger l'oreille pendant des durées très importantes (en pratique au-delà de quelques minutes).

Nous avons vu, par ailleurs, que les cellules ciliées externes contenaient des protéines contractiles et qu'elles étaient innervées par les fibres efférentes (système efférent médian cholinergique) qui commandaient leur contraction (mécanismes lents). L'activation des fibres efférentes en réponse à une stimulation sonore présente une latence de quelques dizaines de millisecondes. Les messages efférents se projettent sur la zone de cochlée qui a donné naissance, quelques dizaines de millisecondes plus tôt, aux messages afférents : il y a là une boucle de rétroaction. La fonction des mécanismes lents est vraisemblablement de contrôler les caractéristiques macro- et micromécaniques de l'organe de Corti. Il y a donc, pour le système nerveux, la possibilité de régler au niveau périphérique le fonctionnement du récepteur auditif et en particulier sa sensibilité au seuil ainsi que sa sélectivité en fréquence [21]. Des études récentes ont montré que, chez le cobaye, les efférences cochléaires pouvaient agir en réduisant les dommages occasionnés par une exposition de la cochlée à des sons intenses [29]. Il peut également se produire une contraction réflexe des CCE induite directement par l'application du stimulus acoustique sur les corps cellulaires. Des études conduites à l'institut Karolinska à Stockholm ont en effet montré que des cellules isolées, excitées acoustiquement in vitro, pouvaient se contracter pour des fréquences bien déterminées [30] sans intervention de la boucle de rétroaction constituée par le système efférent. 
On peut donc penser qu'il existe une sorte de complémentarité entre ces deux réflexes: celui de l'oreille moyenne agissant surtout aux fréquences basses et celui de l'oreille interne aux fréquences élevées.

\section{L'“ entraînement » au bruit}

On considère que l'exposition au bruit n'entraîne aucune habituation et qu'en aucun cas l'oreille ne peut " s'endurcir " et résister de mieux en mieux aux bruits. Cependant Canlon et Borg [31] ont jeté le doute sur cette vérité qui semblait bien établie. Selon ces auteurs, l'importance des déficits auditifs induits par le bruit peut être modifiée de différentes façons. Ils considèrent que les cellules ciliées externes se comportent un peu à la façon de "muscles" et que, en appliquant cette analogie, on peut espérer parvenir à exercer ou " entraîner " la cochlée à tolérer des expositions sonores de plus haut niveau. Dans une étude récente réalisée chez le cobaye, Canlon et Borg ont réussi à réduire les effets lésionnels d'un bruit traumatisant en pré-exposant les cobayes à un stimulus acoustique de bas niveau : le groupe pré-exposé présentait une réduction des déficits auditifs de $20 \mathrm{~dB}$ par rapport au groupe témoin. De plus, ce groupe récupérait totalement après un repos de 2 mois, alors que le groupe témoin continuait à présenter des déficits auditifs de 20 à $30 \mathrm{~dB}$.

Ces résultats montrent que la sensibilité de la cochlée au traumatisme acoustique peut être modifiée par les cellules ciliées externes, mais il faut souligner que le mécanisme responsable de l'effet d' " entraînement " est inconnu et qu'il reste à déterminer le rôle que jouent les muscles de l'oreille moyenne, la circulation sanguine cochléaire, la mécanique de la membrane basiliaire... dans cet effet.

\section{Traumatisme acoustique et régénération?}

Nous ne discuterons pas ici l'efficacité des multiples traitements proposés pour tenter de guérir le traumatisme acoustique ou ses fréquentes conséquences comme les acouphènes, mais nous mettrons l'accent sur un aspect entièrement nouveau de ce problème qui laisse entrevoir l'émergence de nouveaux concepts.

Comme nous l'avons vu plus haut, dans la cochlée des mammiferes, une exposition à un bruit traumatique qui lèse les cellules ciliées cause une perte auditive permanente. Cela est dû à l'impossibilité de produire des cellules ciliées en dehors de la période embryonnaire et donc de remplacer celles qui ont été détruites. A l'opposé, les vertébrés à sang froid ont une production continue de cellules ciliées et peuvent donc remplacer celles qui ont été endommagées. La papille basilaire des oiseaux présente un état intermédiaire : normalement, toutes les cellules ciliées sont produites au cours de l'embryogenèse, mais celles qui sont détruites par traumatisme peuvent être remplacées [32] : les dégâts causés par le bruit stimulent la production de nouvelles cellules ciliées. Les résultats indiquent que dans les 15 heures qui suivent le traumatisme, des cellules situées en bordure inférieure de l'épithélium sensoriel commencent à se diviser et forment, ensuite, de nouvelles cellules ciliées et de nouvelles cellules de soutien. Les nouvelles cellules ciliées se différencient entre 48 et 72 heures : migration vers la surface de l'épithélium et poussée des stéréocils.

Pourquoi ce qui est possible chez les oiseaux ne l'est-il pas chez les mammifères ? S'agit-il de phénomènes métaboliques voire génétiques spécifiques à ces deux classes d'animaux ? Peut-on espérer un jour pénétrer suffisamment ces phénomènes pour entrevoir un début d'application à l'homme?

Voilà un des défis les plus passionnants présenté par l'état actuel des recherches dans le domaine de l'étude des effets des bruits sur l'audition que nous venons de passer en revue.

\section{Conclusion}

L'état de la connaissance des effets des bruits sur l'audition, qui semblait être bien cerné à la fin des années 1970 , s'est trouvé bouleversé par les derniers acquis de la recherche fondamentale : - la découverte des phénomènes cochléaires actifs : oto-émissions, motilité des cellules ciliées de l'organe de Corti, amplificateur cochléaire ; - la mise en évidence de nombreux neuromédiateurs impliqués, au niveau de l'oreille interne, dans les transmissions nerveuses afférentes et efférentes ;

- l'observation de la régénération des cellules ciliées chez les oiseaux après destruction par le bruit ou les ototoxiques...

qui ont relancé de manière spectaculaire les études conduites dans ce domaine.

De nouvelles perspectives se sont ouvertes tant en ce qui concerne la connaissance fondamentale des mécanismes lésionnels des bruits que les possibilités de diagnostic et de traitement de leurs effets ; elles devraient conduire à un véritable bond en avant dans ce domaine de la santé publique au cours de la prochaine décennie

\section{Summary}

The acoustic trauma

The origin of the temporary threshold shifts (TTS) and permanent threshold shifts (PTS) are studied in relation to the physiology and the morphology of the Corti's organ and especially of the outer and inner hair cells. The damages to the stereocilia of the hair cells following exposure to noise seem to be the main factor responsible for the apparition and the extent of the hearing losses. The influence of several parameters : level, duration, frequency..., on TTS and PTS are reviewed and the phenomenon of the half-octave shift is described. The cochlear mechanisms responsible for the threshold shifts seem to be different for the TTS and the PTS. This observation indicates that, for an individual, the TTS observed a few minutes after an acoustic stimula- 


\section{Summary (suite)}

tion does not represent a good prediction of the PTS which could appear after a long term exposure to the same stimulus. Some hypotheses concerning the interindividual and interspecific differences of susceptibility to noise are presented : the conditions according to which the acoustic energy is transmitted from the free field to the inner ear seem to play a very important role. The mechanisms of protection of hearing: the middle ear acoustic reflex and the " intracochlear acoustic reflex " are also responsible for the interindividual differences of susceptibility to noise. The "intracochlear acoustic reflex " could be due to the feedback of the efferent fibers connecting the outer hair cells and/or to the direct mechanical excitation of the outer hair cells by the acoustic stimulus (both mechanisms induce a contraction of these cells and a limitation of their dynamical range). An acoustic stimulus of moderate intensity seems to be able to induce a " training " of the outer hair cells and to diminish the auditory hazards due to the following exposure to a loud noise. Last but not least, new findings obtained in birds indicate that the avian hair cells are able to regenerate after an acoustic trauma ; this finding constitutes a very exciting challenge for the search of new treatments of the cochlear damages.

A. Dancer. 